\title{
Establishment of a Finnish vascular access team
}

\author{
Riku Palanne, MD ${ }^{1}$, Oskar Nyholm, RN, BNSc ${ }^{1}$
}

Affiliations: 1) Peijas Hospital, Department of Anaesthesiology, Intensive Care and Pain Medicine, University of Helsinki and Helsinki University Hospital, Vantaa, Finland

\section{Introduction}

Limiting the number of healthcare professionals who perform vascular catheterizations has many benefits. ${ }^{1,2}$ In Finland physicians have traditionally been responsible for inserting all central venous catheters and managing difficult vascular access (VA).

We started the first Finnish VA team (VAT) in 2015 by educating one nurse to insert midline catheters and peripherally inserted central catheters (PICCs). The objective was to concentrate majority of catheterizations to this nurse and one anesthesiologist and thus improve patient care and reduce costs. Another nurse joined the established VAT in 2017.

\section{Results}

VA nurses are now working independently under supervision of VA anesthesiologist. VAT is responsible for most peripheral catheterizations.

No midline catheter or PICC related bloodstream infections have been reported since $2013 .^{3}$

\section{Education of VA nurses}

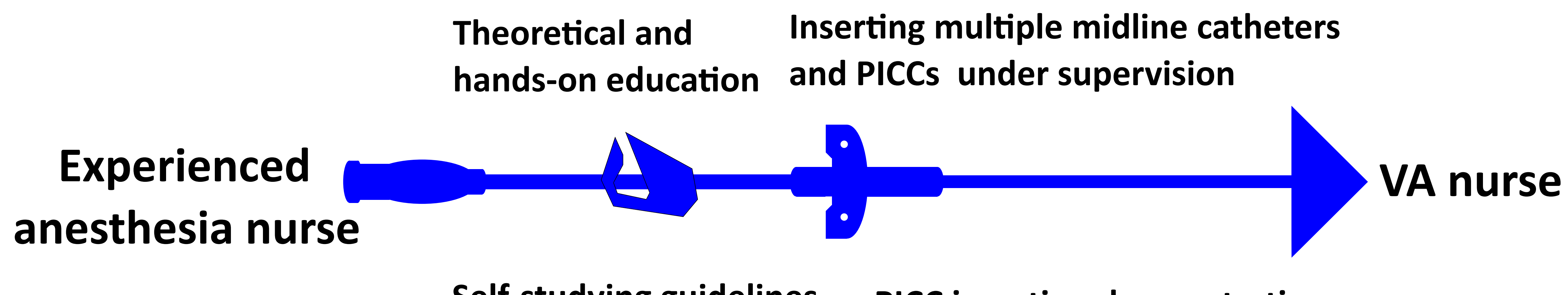

Self-studying guidelines

PICC insertion demonstration and recommendations

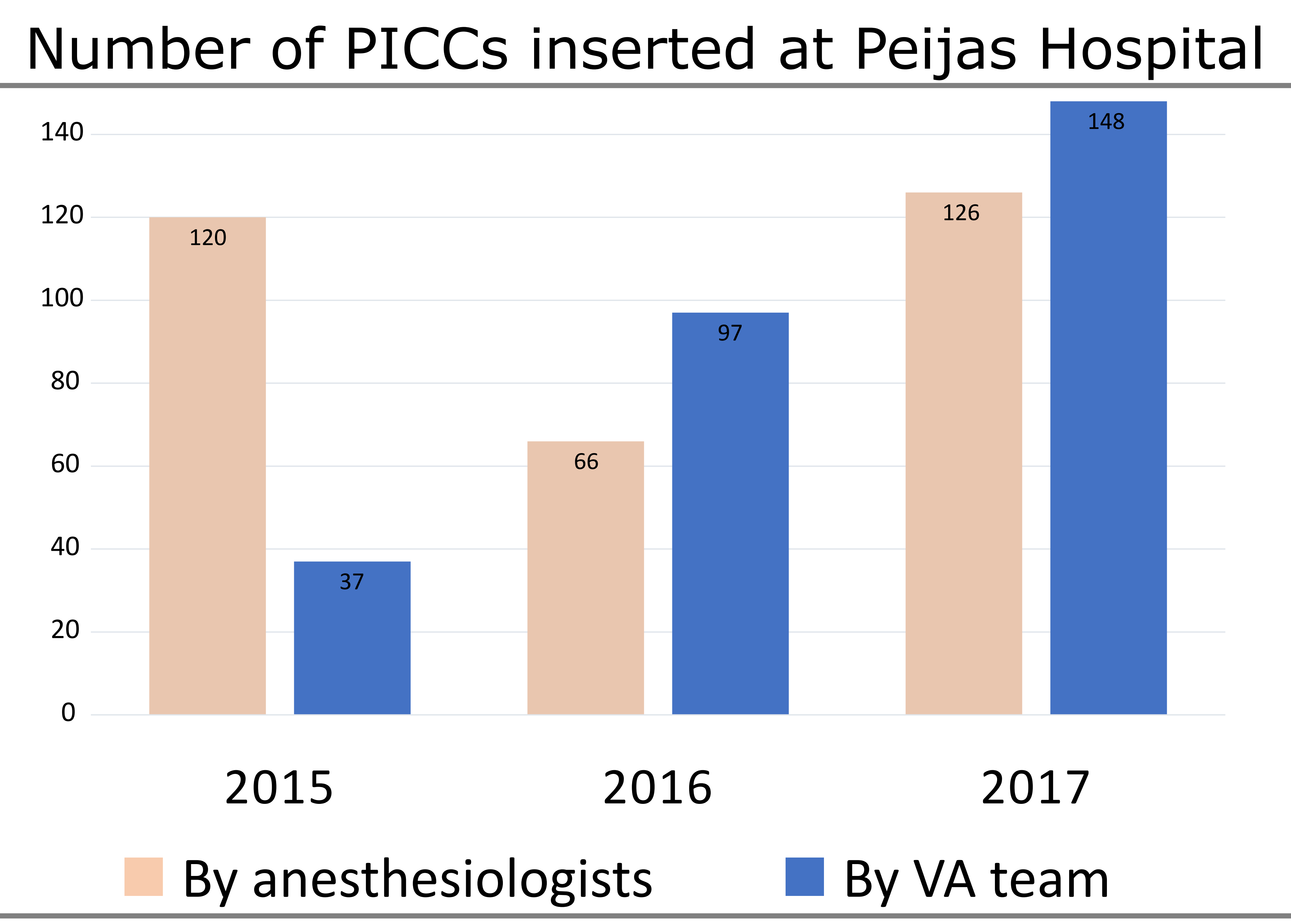

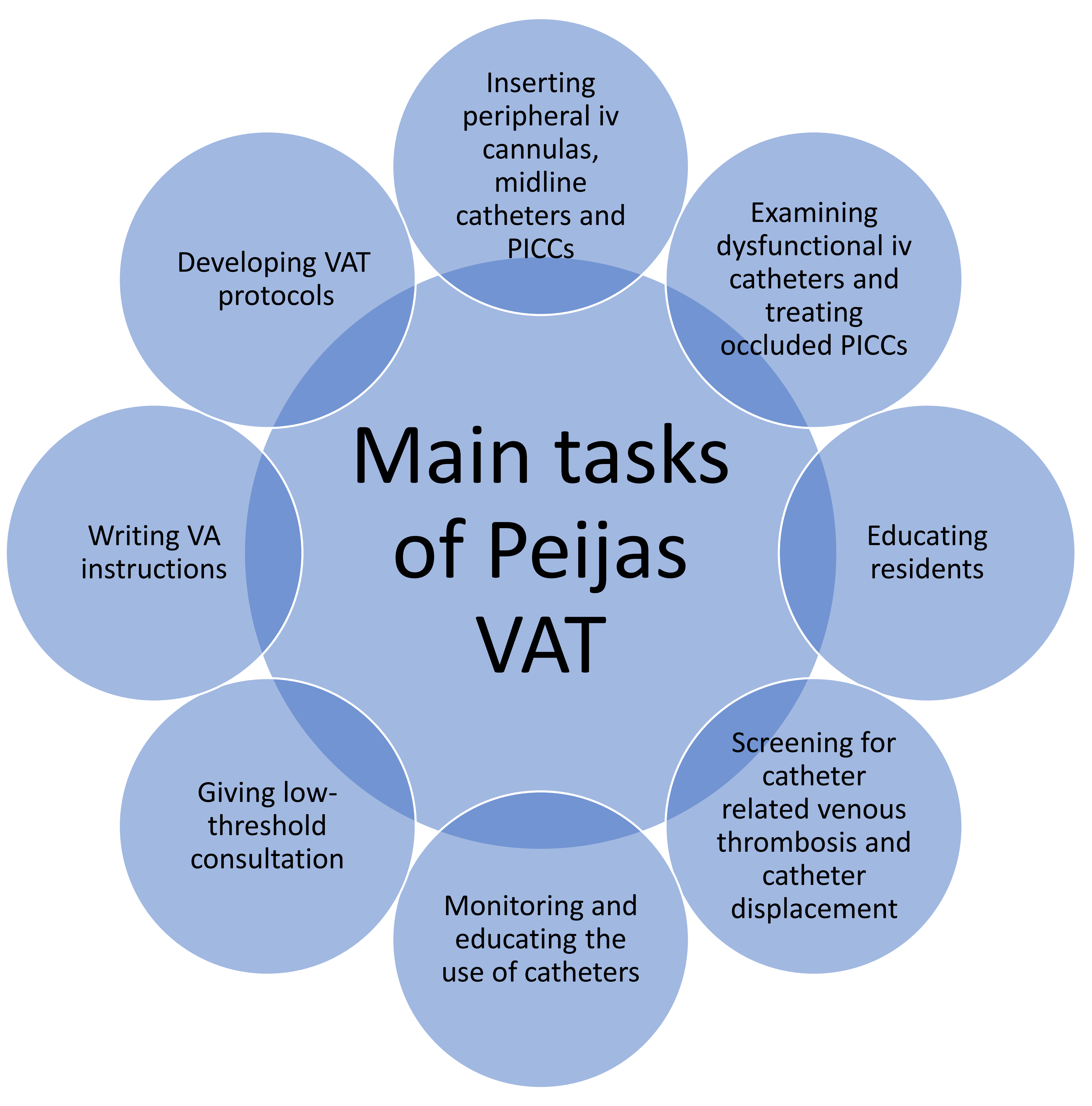

\section{Conclusion and future prospects}

Benefits of having a VAT in our hospital have been recognized. Concentrating all peripheral catheterizations to the team is, however, impossible because Peijas is a teaching hospital.

VA nurses are currently assigned to VA duties 2-3 days in a week. Our next goals are to increase the VA working days and to establish a vascular catheter registry for investigative and quality control purposes.

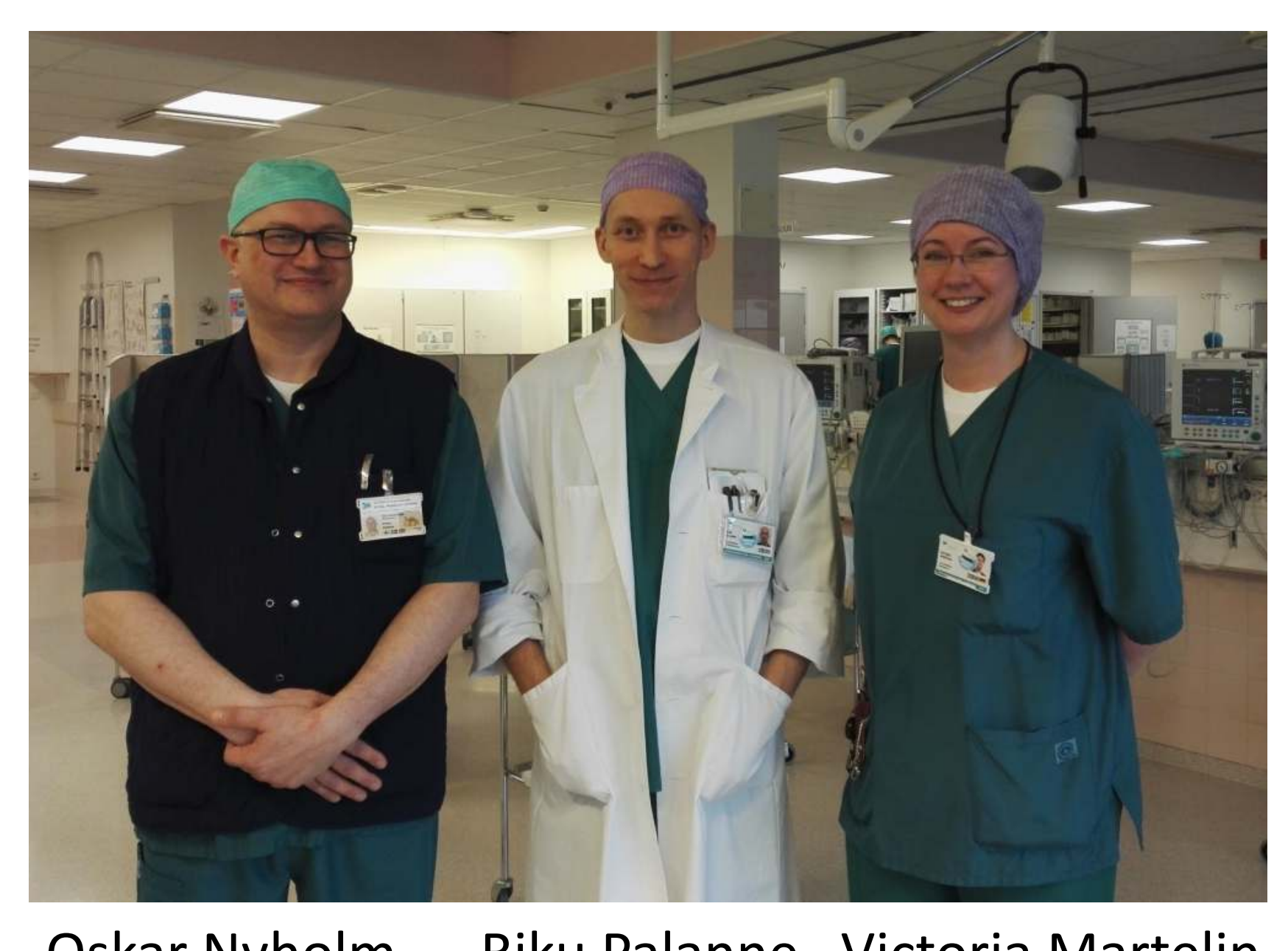

